\title{
Wie wird Energie im Biologieschulbuch dargestellt? - Entwicklung eines Kategoriensystems und exemplarische Anwendung auf eine Schulbuchreihe
}

\author{
Ulrike Wernecke $^{1}$ (D) $\cdot$ Julia Schwanewedel $^{1} \cdot$ Kerstin Schütte $^{2} \cdot$ Ute Harms $^{1}$
}

Eingegangen: 4. April 2016 / Angenommen: 26. September 2016 / Online publiziert: 12. Oktober 2016 (c) Der/die Autor(en) 2016. Dieser Artikel ist eine Open-Access-Publikation.

Zusammenfassung Energie ist ein abstraktes Konzept, das in den Naturwissenschaften eine zentrale Rolle spielt. Zahlreiche Studien zeigen, dass Lernende große Probleme mit dem Verständnis von Energie haben. Deshalb ist fachlich korrektes und potenziell lernförderlich gestaltetes Unterrichtsmaterial zum Thema Energie besonders wichtig. Um die Darstellung von Energie in Medien für den Biologieunterricht inhaltlich und formal zu beschreiben, wurde unter Rückgriff sowohl auf die Repräsentationsforschung als auch auf die Vorstellungsforschung zum Energiekonzept ein Kategoriensystem entwickelt. Anhand dieses Kategoriensystems wurden exemplarisch die energiebezogenen Texte, Abbildungen und Aufgaben in den stoffwechsel- und ökologiebezogenen Kapiteln einer Schulbuchreihe der Sekundarstufe I und II (drei Schulbücher und ein Arbeitsbuch) untersucht. Die Ergebnisse zeigen unter anderem, dass Energieformen und -umwandlungen in den untersuchten Biologieschulbüchern häufig, Energieerhaltung und -entwertung hingegen sehr selten thematisiert werden. Die Abbildungen weisen potenziell lernförderliche Eigenschaften auf. Die

Die Quellen: Bayrhuber et al. (2010); Erdmann et al. (2008); Feldermann (2005) und Konopka et al. (2009) sind Gegenstand der Schulbuchanalyse.

\section{Zusatzmaterial online Zusätzliche Informationen sind in der} Online-Version dieses Artikels (doi: 10.1007/s40573-016-00512) enthalten.

\footnotetext{
Ulrike Wernecke

wernecke@ipn.uni-kiel.de
}

1 Didaktik der Biologie, Leibniz-Institut für die Pädagogik der Naturwissenschaften und Mathematik (IPN), Olshausenstraße 62, 24118 Kiel, Deutschland

2 Erziehungswissenschaft, Leibniz-Institut für die Pädagogik der Naturwissenschaften und Mathematik (IPN), Olshausenstraße 62, 24118 Kiel, Deutschland
Aufgaben könnten durch eine größere Variation in den Anforderungen verbessert werden. Abschließend werden sich aus den Ergebnissen ergebende Forschungsdesiderata aufgezeigt.

Schlüsselwörter Biologie · Energiekonzept · Schulbuchanalyse $\cdot$ Kategoriensystem · Repräsentationen

\section{How is Energy Represented in Biology Textbooks? - Development of a Category System and Its Application to a Textbook Series}

Abstract Energy is an abstract concept which plays a crucial role in science. Numerous studies have shown that students have significant problems understanding energy. Hence, correct and suitable teaching material is especially important. A coding scheme was compiled on the basis of theoretical frameworks of both research on energy conceptions and cognitive psychology to describe the representation of energy in teaching material for biology with regard to content and design. Based on this coding scheme the energy-related texts, pictures and tasks in the metabolism- and ecology-related chapters of a textbook series for lower and upper secondary levels (three textbooks and a workbook) were studied as examples. The results show that whereas forms and transformation of energy are frequently addressed, degradation and conservation are rarely addressed. Pictures in the textbook series were found to employ design features considered beneficial for learning. Tasks could be improved by using a higher variation in the requirements. Based on the results we identify research desiderata. 
Keywords Biology · Category System - Energy Concept . Representations · Textbook Analysis

\section{Einleitung}

Energiewechsel gehört zu den Kennzeichen des Lebendigen. Ohne Energie wäre Leben auf der Erde unmöglich. Folglich spielt Energie als fundamentales Konzept der Biologie für das Verständnis zahlreicher biologischer Themen wie Ernährung, Wachstum oder Bewegung eine zentrale Rolle. Ergebnisse der Vorstellungsforschung zeigen allerdings, dass Schülerinnen und Schüler nur mangelhaftes Wissen über Energie besitzen. Probleme mit dem Verständnis des Energiekonzepts werden seit den 1980er-Jahren immer wieder empirisch belegt (Burger 2001; Duit 1984; Opitz et al. 2015).

Traditionell sind Schulbücher ein zentrales Lehr-Lernmittel im naturwissenschaftlichen Unterricht. Obwohl neue Medien Einzug in den Unterricht gehalten haben, stützen sich Lehrkräfte weiterhin in erheblichem Maße auf das Schulbuch (McDonald 2015). Aus Sicht der Fachdidaktik Biologie ist ein Bedeutungsverlust der Biologieschulbücher zunächst nicht zu erwarten: „Die Bedeutung des Biologie-Schulbuchs dürfte allein aufgrund seiner Verfügbarkeit größer sein als die jedes anderen im Biologieunterricht eingesetzten Mediums." (Gropengießer 2013, S. 390). Physiklehrkräfte verschiedener Schulformen und Bundesländer gaben in einer Befragung an, Schulbücher häufig zur Unterrichtsvorbereitung heranzuziehen. Sie dienen unter anderem zur Auswahl von Inhalten, als Grundlage für Hausaufgaben und als Quelle für Abbildungen (Härtig et al. 2012). In dem Maße, in dem Lehrkräfte Schulbücher für derartige Zwecke heranziehen, werden inhaltliche und didaktische Aspekte des Unterrichts durch das Schulbuch mitbestimmt. Offen ist, inwieweit die Darstellung von Energie in Unterrichtsmedien und speziell in Schulbüchern für das
Fach Biologie das Potenzial hat, den Aufbau eines korrekten Energieverständnisses zu unterstützen. Ziel der hier vorgestellten Studie ist es, ein Kategoriensystem bereit zu stellen, das zur Analyse von Darstellungen des abstrakten Konzepts Energie in Unterrichtsmedien für das Fach Biologie genutzt werden kann und es exemplarisch auf eine vielfach genutzte Schulbuchreihe anzuwenden.

\section{Das Energiekonzept und seine Darstellung in Unterrichtsmedien}

Die Einführung des Energiekonzepts erfolgt traditionell im Physikunterricht (KMK 2004b). In der Physik herrscht Konsens darüber, dass es keine Definition von Energie gibt, die in allen Kontexten gültig ist. $\mathrm{Zu}$ fassen ist lediglich eine abstrakte Größe, die berechnet werden kann (Feynman et al. 2007). Eine Empfehlung aus der Physikdidaktik lautet daher, in Lehr-Lern-Kontexten keine Definition von Energie zu geben (Nordine et al. 2011; Trumper 1991). Das physikalische Energiekonzept wird anhand von vier Aspekten strukturiert (Duit 1984; Neumann et al. 2013) und lässt sich auch auf biologische Themen anwenden (Opitz et al. 2015; vgl. Tab. 1). Zwar stellt Energie in den Bildungsstandards im Fach Biologie für den Mittleren Schulabschluss - anders als in den Bildungsstandards der Fächer Chemie und Physik - kein eigenes Basiskonzept dar, es wird jedoch maßgeblich zur Beschreibung des Basiskonzepts System herangezogen: Stoff- und Energieumwandlungen sind Eigenschaften lebendiger Systeme auf Zell- und Organismusebene, Energiefluss wird als eine Eigenschaft des Ökosystems und der Biosphäre beschrieben (KMK 2004a). Ein Verständnis der Themenbereiche Stoffwechsel und Ökologie erfordert eine Betrachtung des Energiekonzepts auf unterschiedlichen biologischen Systemebenen.

Duit (2014) argumentiert, dass jeder der vier Energieaspekte nur im Zusammenhang mit den jeweils anderen drei-

Tab. 1 Energieaspekte und Beispiele für Alternativvorstellungen

\begin{tabular}{lll}
\hline Energieaspekt & Fachliches Beispiel & $\begin{array}{l}\text { Beispiel für eine analoge Alternativvorstel- } \\
\text { lung von Schülerinnen und Schülern in der } \\
\text { Biologie (Burger 2001) }\end{array}$ \\
\hline Energieformen und -quellen & $\begin{array}{l}\text { Energie tritt in verschiedenen Formen auf. Die ursprüngli- } \\
\text { che Energiequelle für die meisten Ökosysteme ist die Sonne }\end{array}$ & $\begin{array}{l}\text { Pflanzen nehmen Energie aus dem Boden } \\
\text { auf }\end{array}$ \\
$\begin{array}{l}\text { Energieumwandlung und -über- } \\
\text { tragung }\end{array}$ & $\begin{array}{l}\text { Bei Stoffwechselprozessen wird eine Energieform in eine } \\
\text { oder mehrere andere Energieformen umgewandelt. Energie } \\
\text { kann von einem System auf ein anderes übertragen werden } \\
\text { Energieentwertung }\end{array}$ & $\begin{array}{l}\text { Energie kann direkt in ,etwas“ umgewan- } \\
\text { delt werden, z. B. in Muskeln oder Fett }\end{array}$ \\
& $\begin{array}{l}\text { Bei jedem Energieumwandlungsprozess wird Wärmeener- } \\
\text { gie frei, die an die Umgebung abgegeben wird. Diese Wär- } \\
\text { meenergie kann von Lebewesen nicht mehr in andere Ener- } \\
\text { gieformen umgewandelt werden }\end{array}$ & $\begin{array}{l}\text { Lebewesen nehmen Energie für bestimmte } \\
\text { (genannte) Lebensvorgänge in Form von } \\
\text { Wärme auf }\end{array}$ \\
& $\begin{array}{l}\text { Die Gesamtenergie im Universum ist immer gleich, denn } \\
\text { Energie kann nicht erzeugt oder zerstört werden }\end{array}$ & $\begin{array}{l}\text { Energie kann hergestellt (z. B. aus Nah- } \\
\text { rung) und (z. B. für Lebensvorgänge) ver- } \\
\text { braucht werden }\end{array}$ \\
\hline Energieerhaltung & &
\end{tabular}


en verstanden werden kann. Deshalb sollte der naturwissenschaftliche Unterricht alle vier Aspekte des Energiekonzepts berücksichtigen (Lacy et al. 2014). Zahlreiche Studien der Vorstellungsforschung haben gezeigt, dass Schülerinnen und Schüler aller Klassenstufen und Schulformen Probleme mit dem Verständnis des Energiekonzepts haben. Vor allem das Verständnis der Aspekte Energieerhaltung und -entwertung ist gering (Duit 1984; Jin und Anderson 2012; Neumann et al. 2013). Zudem konnte die Existenz von zahlreichen Alternativvorstellungen nachgewiesen werden (für die Biologie Burger (2001); vgl. Tab. 1). Umgangssprachlich wird das Wort Energie anders verwendet als in der Fachsprache. Beispielsweise wird in der Alltagssprache Energie mit Leben und Vitalität assoziiert: So hat man etwa nach einer erholsamen Nacht besonders viel Energie für den nächsten Tag (Jin und Wei 2014). Im Sinne einer konstruktivistischen Auffassung von Lernen sollten Alternativvorstellungen der Schülerinnen und Schüler als Ausgangspunkt des Lernens berücksichtigt werden, indem beispielsweise an sie angeknüpft wird oder ein Kontrast zur wissenschaftlich korrekten Vorstellung aufgebaut wird (z. B. Özkan et al. 2004; Trumper 1991).

Trotz der bekannten Verständnisprobleme gibt es bislang nur wenige Analysen, die sich mit der inhaltlichen oder formalen Darstellung von Energie im Unterrichtsmedium Schulbuch beschäftigen. Ein Großteil davon ist in der Physik verortet und fokussiert auf ein bestimmtes energiebezogenes Thema (z. B. Ibánez und Ramos 2004). Im Fach Biologie existiert unseres Wissens nach nur eine Arbeit von Opitz et al. (2015), in der die Häufigkeit der Thematisierung von Energie in neun Biologieschulbüchern von der Grundschule bis zur Sekundarstufe II untersucht wurde. Insgesamt nahm die Anzahl der Nennungen der Energieaspekte mit steigender Klassenstufe zu. Die Aspekte Energieerhaltung und -entwertung machten nur 10-16\% der Thematisierungen aus.

\section{Lernen mit externen Repräsentationen in Unterrichtsmedien}

Welche Bedeutung haben Repräsentationen für den Erwerb eines angemessenen Energiekonzepts? Repräsentationen sind Zeichen, sie stehen also stellvertretend für etwas Bezeichnetes. $\mathrm{Zu}$ unterscheiden sind interne (mentale) und externe Repräsentationen. Letztere werden in deskriptive (basierend auf Symbolen, z. B. Texte) und depiktive (basierend auf ikonischen Zeichen, z. B. Piktogramme) Repräsentationen unterteilt (nach Schnotz 2002). Im Folgenden sprechen wir zur Verbesserung der Anschaulichkeit vereinfachend von Texten bzw. Abbildungen. Das Verstehen einer externen Repräsentation ist ein aktiver Informationsverarbeitungsprozess. Dabei baut das Individuum eine interne, mentale Repräsentation des sprachlich beschriebenen oder bildlich gezeigten Gegenstands auf, die in bereits vorhandene Vorstellungen integriert wird (Schnotz und Bannert 2003). Anders als in der holistischen Betrachtungsweise, die Repräsentationen als Fachsprache ansieht (vgl. u.a. Nitz et al. 2012), wird Fachsprache hier umgekehrt als fachspezifische Ausprägung der Unterrichtssprache (Härtig et al. 2015) und somit als ein Teil von Repräsentationen verstanden. Die Fähigkeit, Informationen aus Repräsentationen zu erschließen und diese auszutauschen, kann als integraler Teil fachspezifischer Kommunikationskompetenz im Sinne einer erweiterten Lese- und Verstehenskompetenz aufgefasst werden, welche durch die Bildungsstandards im Fach Biologie für den Mittleren Schulabschluss gefordert wird (KMK 2004a).

Grundsätzlich wird davon ausgegangen, dass das Lernen mit Bild-Text-Kombinationen zu größeren Lerneffekten führt als Lernen mit Texten allein (Multimedia principle, Mayer 2011). Abbildungen können das Verständnis abstrakter Konzepte unterstützen, indem sie für das menschliche Auge nicht wahrnehmbare Phänomene visualisieren (Tsui und Treagust 2013). Fotos und realistische Zeichnungen sind darstellende, Graphen und Diagramme hingegen logisch-analytische Abbildungen (Weidenmann 2004). Auszählungen bei naturwissenschaftlichen Schulbüchern verschiedener Klassenstufen und Länder haben ergeben, dass pro Seite durchschnittlich eineinhalb bis zwei Abbildungen vorhanden sind (z. B. Liu und Treagust 2013; Mayer 1993), wobei die Bilddichte mit steigender Klassenstufe abnimmt (Dimopoulos et al. 2003).

Die Lernwirksamkeit von Abbildungen ist von deren Gestaltungsmerkmalen und der kognitiven Auseinandersetzung der Lernenden mit der Abbildung abhängig (Ainsworth 2006; Cromley et al. 2010). Logisch-analytische Abbildungen stellen höhere kognitive Anforderungen an Lernende als darstellende Bilder, da sie keine Ähnlichkeit mit dem abzubildenden Objekt besitzen (Schnotz 1994). Der Großteil der verwendeten Abbildungen in naturwissenschaftlichen Schulbüchern ist darstellenden Typs (Roth et al. 1999; Slough et al. 2010), wobei der Anteil logisch-analytischer Abbildungen mit steigender Klassenstufe zunimmt (Dimopoulos et al. 2003; Liu und Treagust 2013). Vielfach wurde auf die lernförderliche Wirkung von Untertiteln und Beschriftungen bei logisch-analytischen naturwissenschaftlichen Abbildungen hingewiesen (für das Thema Energie: Ametller und Pintó (2002)).

Für die Bewertung von Schulbüchern ist neben der Art der Abbildung auch deren Funktion essentiell (Slough und McTigue 2013). Die funktionale Taxonomie von Levin et al. (1987) stellt hierfür bis heute das gängigste Klassifikationssystem dar (Slough und McTigue 2013). Sie unterscheidet vier Funktionen von Abbildungen für Schulbücher: (1) Dekoration, (2) Repräsentation, (3) Organisation und (4) Inter- 
pretation. Eine dekorative Abbildung dient lediglich dazu, die Attraktivität eines Textes zu erhöhen. Repräsentative Abbildungen konkretisieren den Text, indem sie ein wesentliches Element aus diesem darstellen. Abbildungen mit Organisationsfunktion zeigen die logische Organisation des Textinhalts und machen den Text stimmiger, indem sie z. B. Verbindungen zwischen Objekten zeigen. Eine Abbildung mit Interpretationsfunktion geht darüber hinaus, indem sie besonders schwierige Textpassagen verdeutlicht und den Text verständlicher macht. Levin et al. (1987) schränken ein, dass sich die Funktionen gegenseitig nicht zwingend ausschließen und vor allem die Interpretationsfunktion vom Wissensstand der Lernenden abhängig ist. Zwischen Organisations- und Interpretationsfunktion wird in Schulbuchanalysen nicht immer getrennt (Slough et al. 2010). In einer Metaanalyse mit 150 Studien zeigte sich, dass dekorative Bilder leicht negativ auf den Lernerfolg wirken, während die Funktionen (2) bis (4) zunehmend große positive Effekte auf den Lernerfolg aufweisen (Levin et al. 1987). Nichtsdestoweniger sind keine pauschalen Aussagen über die Lernwirksamkeit möglich. Untersuchungen von naturwissenschaftlichen Schulbüchern haben ergeben, dass Abbildungen mit repräsentativer Funktion mit über 50\% am häufigsten auftreten, gefolgt von dekorativen Abbildungen, während organisierende Abbildungen mit unter $10 \%$ am wenigsten vorkommen (Mayer 1993; Slough et al. 2010).

Das Verhältnis zwischen Text und Abbildung sollte nicht nur funktional, sondern auch inhaltlich betrachtet werden. Die Informationen mehrerer Repräsentationen können sich mehr oder weniger stark überlappen. Die Relation ist dabei eng verknüpft mit der Funktion der Abbildung: In der Regel sind dekorative und repräsentative Abbildungen in einem höheren Maße redundant als Abbildungen mit organisatorischer oder interpretativer Funktion. Überlappungen zwischen Text- und Bildinformationen können für Lernende hilfreich sein, zum Beispiel wenn die Interpretation eines neuen Abbildungstyps gelernt werden soll (Ainsworth 1999; Kalyuga et al. 1998). Redundanzen erhöhen allerdings die Belastung des Arbeitsgedächtnisses (Sweller 2005).

Das adäquate Design des Unterrichtsmaterials ist für effektives Lernen mit Texten und Abbildungen notwendig, aber nicht hinreichend, denn auch die Aufforderung zum Lernen (kognitive Aktivierung) muss angemessen sein. Aufgaben können Schülerinnen und Schüler dazu anregen, Abbildungen und Texte zu verarbeiten und eigene mentale Modelle des dargestellten Inhalts zu generieren (Bodemer und Faust 2006). Am deutschsprachigen Biologieunterricht wird aber die Dominanz von Aufgaben mit geringen kognitiven Anforderungen kritisiert (z. B. Maier et al. 2010).

Das Lernen mit Repräsentationen kann durch Informationsentnahme, Konstruktion und Integration erfolgen (für Diagramme: Lachmayer et al. (2007)). Bei der Informati- onsentnahme erschließen Lernende Informationen aus einem Text oder einer Abbildung. Bei der Konstruktion müssen Lernende einen Text oder eine Abbildung ergänzen oder vollständig neu erstellen. Das Verknüpfen von Informationen aus mehreren Repräsentationen wird als Integration bezeichnet (Maier et al. 2010). Integrationsaufgaben können den Lernerfolg erhöhen, sind aber besonders für Lernende mit geringem Vorwissen schwierig (Ainsworth 1999; Bodemer und Faust 2006).

\section{Fragestellung}

Biologieschulbücher müssen fachlich korrekte Repräsentationen enthalten, die Schülerinnen und Schüler unterstützen können, ein Verständnis des naturwissenschaftlichen Basiskonzepts Energie aufzubauen. Abgesehen von der Auszählung, wie häufig Energieaspekte thematisiert wurden (Opitz et al. 2015), gibt es unseres Wissens bislang keine systematische Analyse zur Darstellung des Energiekonzepts in Biologieschulbüchern. Auch fehlt hierfür ein geeignetes, energiespezifisches Kategoriensystem. Ziel der vorliegenden Studie ist es daher, zunächst ein Kategoriensystem zur Analyse der Darstellung von Energie in Medien für den Biologieunterricht zu entwickeln. Im zweiten Schritt wurde dieses Kategoriensystem genutzt, um exemplarische Erkenntnisse über die inhaltliche und die formale Gestaltung energiebezogener Texte, Abbildungen und Aufgaben in Medien für den Biologieunterricht zu erarbeiten. Durch die Analyse einer Schulbuchreihe sollen erste Informationen darüber gewonnen werden, welche Unterschiede in der inhaltlichen und formalen Darstellung des Energiekonzepts in Schulbüchern der Sekundarstufe I und II bestehen.

\section{Methode}

In der Regel unterliegen Schulbücher einem staatlichen Genehmigungsverfahren. ${ }^{1}$ Für die Repräsentationsanalyse wurde die Linder-Reihe des Schroedel-Verlags ausgewählt, von der in jedem Bundesland mindestens zwei Bände zugelassen sind (Stand: 2015). In der Sekundarstufe I darf der Linder in dreizehn Bundesländern ${ }^{2}$ verwendet werden, in der Sekundarstufe II in allen sechzehn Bundesländern. ${ }^{3}$

\footnotetext{
${ }^{1}$ In fünf Bundesländern (Berlin, Brandenburg, Hamburg, Saarland und Schleswig-Holstein) gibt es kein zentrales Zulassungsverfahren für (Biologie-)Schulbücher und Lernmittel. Quellen siehe KMK (2015).

2 In fünf Bundesländern (Baden-Württemberg, Mecklenburg-Vorpommern, Nordrhein-Westfalen, Sachsen, Thüringen) sind für die Sekundarstufe I nur die bundeslandspezifischen Ausgaben zugelassen.

${ }^{3}$ In Bayern sind nur die bundeslandspezifischen Bände für die Sekundarstufe II zugelassen.
} 
In der Studie wurden drei Bände analysiert, die von unterschiedlichen Autorengruppen verfasst wurden: Linder Biologie 1 für die 5. und 6. Klassenstufe (Erdmann et al. 2008), Linder Biologie 2 für die 7. bis 10. Klassenstufe (Konopka et al. 2009) und der Linder Biologie Gesamtband für die Sekundarstufe II (Bayrhuber et al. 2010). Es wurde jeweils die länderübergreifende Ausgabe verwendet. Um die Stichprobe der Aufgaben zu erhöhen, wurde das Linder Biologie Arbeitsbuch ${ }^{4}$ (Feldermann 2005) für die Sekundarstufe II hinzugezogen.

Schulbuchanalysen folgen den Grundsätzen der Inhaltsanalyse (Wang 1998): Grundlegendes Element ist die theoretisch fundierte Erarbeitung eines Kategoriensystems und die Erstellung eines Kodierleitfadens. Zur Überprüfung der Interraterreliabilität sollten mindestens zwei unabhängige Rater eingesetzt und ein Übereinstimmungskoeffizient berechnet werden. Dies dient der Qualitätsüberprüfung der gewonnenen Daten und des Codierleitfadens. Im Rahmen dieser Anforderungen sind unterschiedliche methodische Umsetzungen möglich (vgl. z. B. Stern und Roseman (2004) zur Schulbuchbewertung anhand einer Ordinalskala und Slough \& McTigue (2013) zur Erstellung eines hauptsächlich nominalskalierten Kategoriensystems zur Beschreibung von Texten und Abbildungen).

Um die Texte, Abbildungen und Aufgaben auszuwählen, welche als energiebezogen anzusehen sind, wurde in vier Schritten vorgegangen (vgl. auch Onlinematerial 1). Die Analyse beschränkte sich auf die Themenbereiche Stoffwechsel und Ökologie, in denen das Thema Energie auf verschiedenen biologischen Systemebenen betrachtet wird. Entsprechend wurden in einem ersten Schritt die dazugehörigen Kapitel ausgewählt. Die Kapitel bestehen aus Unterkapiteln, die durchschnittlich 1,6 bis 1,8 Seiten umfassen. Der Text eines Unterkapitels wurde als eine Analyseeinheit betrachtet. In einem zweiten Schritt wurden die Unterkapitel ausgewählt, in denen das Wort Energie oder ein dazugehöriges Kompositum in Text oder Abbildung mindestens einmal vorhanden ist. Innerhalb dieser Unterkapitel wurden in einem dritten Schritt die energiebezogenen Texte und Abbildungen ausgewählt. Der vierte Schritt stellte die Auswahl der energiebezogenen Aufgaben dar. Innerhalb der ökologischen bzw. stoffwechselbiologischen Kapitel wurden nur die Aufgaben analysiert, in denen das Wort Energie oder ein dazugehöriges Kompositum in Aufgabenstamm und/oder in der Musterlösung der Lösungsbände vorkommt. Das Auswahlverfahren ergab $N=525$ Analyseeinheiten: 103 Texte,

\footnotetext{
${ }^{4}$ Das Linder Arbeitsbuch bezieht sich auf die 22. Auflage des Linder
} Gesamtbandes, da zur aktuellen 23. Auflage keines erschienen ist.
182 Abbildungen für die Fließtextanalyse und 127 Aufgaben mit 113 Abbildungen für die Aufgabenanalyse. ${ }^{5}$

Im Rahmen der Fließtextanalyse wurden Texte und Abbildungen der Unterkapitel einer inhaltlichen und formalen Analyse unterzogen. In einem deduktiven Verfahren wurden Kategorien aus der Literatur abgeleitet. Als Grundlage für die inhaltliche Analyse dienten Studien zur Vorstellungsforschung (z. B. Burger 2001; Duit 1984) und die theoretische Beschreibung des Energiekonzepts (z. B. Duit 2014). Ausgangspunkt für die formale Analyse waren v. a. die im Theorieteil genannten konzeptuellen und empirischen Forschungsarbeiten zur Gestaltung von Abbildungen. Bei der Aufgabenanalyse wurden die Aufgabenstellungen sowie die Texte und Abbildungen, auf die sich die Aufgaben beziehen, inhaltlich und formal betrachtet. Die Lösungsbände wurden verwendet, um die fachlichen und kognitiven Anforderungen, die die jeweilige Aufgabe stellt, einzuschätzen. Im Linder 1 befinden sich $40,0 \%$ und im Linder 2 $69,0 \%$ der energiebezogenen Aufgaben in Unterkapiteln und nicht auf gesonderten Aufgabenseiten. Deshalb sind einige Abbildungen, die schon bei der Fließtextanalyse untersucht wurden, auch in die Aufgabenanalyse eingeflossen. Im Linder Gesamtband hingegen befinden sich Aufgaben und die dazugehörenden Abbildungen auf Aufgabenseiten. Das Arbeitsbuch besteht ausschließlich aus Aufgaben. Deshalb gab es bei diesen beiden Bänden keine Überlappungen von Fließtext- und Aufgabenanalyse.

Um die Güte des Auswahlverfahrens der energiebezogenen Repräsentationen sicherzustellen, wurden von den stoffwechsel- und ökologiebezogenen Unterkapiteln $(N=291) 25 \%$ jedes Bandes zufällig ausgewählt. Sie bestanden aus $N=385$ Texten und Abbildungen, die von dem zweiten Rater hinsichtlich der Existenz eines Energiebezugs bewertet wurden. Die Berechnung des KappaKoeffizienten nach Cohen (1960) als Maß für die Interraterreliabilität ergab $\kappa=0,72^{6}$. Laut Landis und Koch (1977) zeigt dieser Wert eine substanzielle Übereinstimmung zwischen den Ratern an. Bei der Auswahl der energiebezogenen Aufgaben wurde ein eindeutiges Kriterium angelegt (Nennung des Wortes Energie oder Komposita im Aufgabenstamm und/oder in der Musterlösung), sodass auf eine Überprüfung der Interraterreliabilität verzichtet werden konnte.

\footnotetext{
5 In Linder 1 und in Linder 2 sind einige Abbildungen, die bei der Fließtextanalyse untersucht wurden, auch in die Aufgabenanalyse eingeflossen. Es handelt sich insgesamt um 499 verschiedene Analyseeinheiten.

${ }^{6}$ Nicht-Übereinstimmungen zwischen den Ratern bei dem Auswahlverfahren der energiebezogenen Repräsentationen liegen vermutlich darin begründet, dass es sich bei dem Energiebezug um ein Kontinuum handelt, da Energie als abstraktes Konzept unterschiedlich deutlich im Material angesprochen wird. In die Analyse sind auch Materialien eingeflossen, die einen geringen Energiebezug aufweisen.
} 


\section{Ergebnisse der Erarbeitung und Überprüfung des Kategoriensystems}

Das Kategoriensystem wird in Tab. 2 dargestellt.

Die Kriterien für die einzelnen Kategorien sind im Detail im Kodierleitfaden (Onlinematerial 2) beschrieben. An dieser Stelle sollen nur die für die Interpretation der gewonnenen Ergebnisse wichtigsten Kriterien aufgeführt werden:

1. Bei der Zuordnung zu der Kategorie „Thematisierte Aspekte des Energiekonzepts" sind Mehrfachzuordnungen möglich. Es wird zwischen expliziten und impliziten Thematisierungen unterschieden:

Die Thematisierung der Energieform gilt als explizit, wenn eine Energieform oder ein Energieträger/-speicher/-quelle als solche/r benannt wird. Bei einer impliziten Thematisierung wird kein direkter Zusammenhang zu Energie hergestellt, sondern nur ein Energieträger, -speicher oder -quelle genannt oder dargestellt, z. B. die Sonne.

Energieumwandlung/-übertragung ist explizit, wenn der Begriff Energie oder Komposita in Zusammenhang mit einem Wort aus dem Bereich Übertragung, Umwandlung, Transport, Aufbau, Abbau o. ä. steht. Eine implizite Thematisierung liegt vor, wenn das Wort Energie oder Komposita nicht genannt oder dargestellt werden, es sich aber trotzdem um Energieumwandlungs- oder Übertragungsprozesse handelt. Schlagworte wie Energieeinsatz oder Energiegewinnung sind lediglich implizite Thematisierungen von Energieumwandlung, solange sie nicht näher erläutert werden.

Energieentwertung wird nur dann explizit thematisiert, wenn deutlich gemacht wird, dass Energie in Form von (Ab-)Wärme für das System verloren geht bzw. sich der
Nutzen der Energie verringert. Wärmeabgabe stellt lediglich eine implizite Thematisierung dar.

Energieerhaltung gilt als explizit, wenn erklärt wird, dass Energie weder erzeugt noch vernichtet werden kann bzw. dass der Betrag der Energie vor und nach einer Energieumwandlung gleich ist. Bei der impliziten Thematisierung wird dies umschrieben.

Die Thematisierung in Abbildungen wird mit Blick auf den dazugehörenden Textabschnitt bewertet. Sobald eine explizite Nennung auftritt, wird der gesamte Text bzw. die gesamte Abbildung in diesem Aspekt explizit, sodass implizite Nennungen dieses Aspektes nicht mehr gezählt werden.

2. Hinsichtlich der Funktionen von Abbildungen nach Levin et al. (1987) konnten Organisation und Interpretation empirisch nicht getrennt werden (keine ausreichende Interraterübereinstimmung) und wurden deshalb zusammengefasst. Über die Funktionen von Levin et al. (1987) hinausgehend wurde für diese Studie die Funktion $\mathrm{Zu}$ satz definiert. Bei dieser Funktion dient die Abbildung in erster Linie dazu, Zusatzinformationen oder Beispiele darzustellen, die nicht im Text genannt werden.

3. Die Kategorie Relation zeigt den Grad der inhaltlichen Redundanz von Abbildungs- und Textinformation und steht damit im engen Zusammenhang zu der Funktion der Abbildung. Die stark redundante Relation liegt vor, wenn alle Informationen, die in der Abbildung dargestellt sind, auch im Text vorhanden sind. Bei einer schwach redundanten Relation beinhalten Text und Abbildung zum Teil dieselben und zum Teil nur einmal auftretende Informationen. Die Relation ergänzend bedeutet, dass keine substanzielle Überlappung zwischen den präsentierten Informationen besteht.

Tab. 2 Grundlegendes Kategoriensystem und Anwendungsbereich der Kategorien

\begin{tabular}{|c|c|c|c|}
\hline & Kategorie & Beschreibung & Anwendung \\
\hline \multirow[t]{3}{*}{ Inhaltlich } & Definition von Energie & Gegeben oder nicht gegeben & $\mathrm{F}$ \\
\hline & Alternativvorstellungen & $\begin{array}{l}\text { Darstellung oder Aufgreifen von Alternativ- } \\
\text { vorstellungen }\end{array}$ & $\mathrm{F}, \mathrm{A}$ \\
\hline & $\begin{array}{l}\text { Thematisierte Aspekte des Energiekon- } \\
\text { zepts }\end{array}$ & $\begin{array}{l}\text { Form/Speicher, Umwandlung/Übertragung, } \\
\text { Entwertung, Erhaltung }\end{array}$ & $\mathrm{F}, \mathrm{A}$ \\
\hline \multirow[t]{6}{*}{ Formal } & Abbildungstyp & Tabelle, Foto usw & $\mathrm{F}, \mathrm{A}$ \\
\hline & Gestaltung der Abbildungen & Beschriftung, Untertitel & $\mathrm{F}, \mathrm{A}$ \\
\hline & $\begin{array}{l}\text { Funktion der Abbildung in Bezug auf den } \\
\text { Text }\end{array}$ & $\begin{array}{l}\text { Dekoration, Repräsentation, Organisation/ } \\
\text { Interpretation, Zusatz }\end{array}$ & $\mathrm{F}$ \\
\hline & Relation von Abbildung und Text & $\begin{array}{l}\text { Schwach redundant, stark redundant oder } \\
\text { ergänzend }\end{array}$ & $\mathrm{F}$ \\
\hline & Funktion der Abbildung für die Aufgabe & $\begin{array}{l}\text { Dekoration, Vorlage, Informationsbereitstel- } \\
\text { lung }\end{array}$ & A \\
\hline & Anforderungen der Aufgabe & $\begin{array}{l}\text { Informationsentnahme, Konstruktion, Inte- } \\
\text { gration, Fachsprache }\end{array}$ & A \\
\hline
\end{tabular}

$F$ Anwendung der Kategorie bei der Analyse des Fließtextes einschließlich seiner Abbildungen, A Anwendung der Kategorie bei der Aufgabenanalyse 
Tab. 3 Häufigkeit der Thematisierung von Energie in Kapiteln zu Stoffwechselbiologie und Ökologie

\begin{tabular}{|c|c|c|c|c|}
\hline & Linder 1 & Linder 2 & Linder Gesamtband & $\begin{array}{l}\text { Linder Arbeits- } \\
\text { buch }\end{array}$ \\
\hline $\begin{array}{l}\text { Anteil der Unterkapitel, die einen Energie- } \\
\text { bezug aufweisen }\end{array}$ & $\begin{array}{l}17,6 \% \\
\text { (34 Seiten) }\end{array}$ & $\begin{array}{l}33,6 \% \\
(64 \text { Seiten })\end{array}$ & $\begin{array}{l}63,2 \% \\
(79 \text { Seiten) }\end{array}$ & - \\
\hline $\begin{array}{l}\text { Explizite Nennungen des Begriffs Energie } \\
\text { oder Komposita (Fließtextanalyse) }\end{array}$ & 104 & 243 & 319 & - \\
\hline $\begin{array}{l}\text { Durchschnittliche Anzahl der Nennungen } \\
\text { von Energie oder Komposita pro Text/ } \\
\text { Abbildung }\end{array}$ & $\begin{array}{l}M=1,86 \\
S D=4,81\end{array}$ & $\begin{array}{l}M=2,51 \\
S D=5,64\end{array}$ & $\begin{array}{l}M=2,42 \\
S D=5,48\end{array}$ & - \\
\hline Anteil der Aufgaben mit Energiebezug & $\begin{array}{l}4,8 \% \\
N=20\end{array}$ & $\begin{array}{l}8,0 \% \\
N=42\end{array}$ & $\begin{array}{l}16,4 \% \\
N=18\end{array}$ & $\begin{array}{l}13,7 \% \\
N=47\end{array}$ \\
\hline
\end{tabular}

Der zweite Rater kategorisierte je zufällig ausgewählte $25 \%$ der energiebezogenen Texte, Abbildungen und Aufgaben. Bei der Fließtextanalyse bewegen sich die Werte der Interraterübereinstimmung für die einzelnen Kategorien zwischen $\kappa=0,57$ (moderate Übereinstimmung) und $\kappa=1$ (perfekte Übereinstimmung), bei der Aufgabenanalyse zwischen $\kappa=0,64$ (substanzielle Übereinstimmung) und $\kappa=1$ (Beurteilung der Werte nach Landis und Koch [1977]). Nicht mit einbezogen sind dabei die Kategorien, die ein sogenanntes Kappa-Paradox, also sehr niedrige Kappa-Werte trotz einer hohen prozentualen Übereinstimmung beider Rater ( $\geq 75 \%$ ), aufweisen. Es tritt auf, wenn die Verteilung der Beobachtungen auf die möglichen Kategorien stark ungleich ist (vgl. Onlinematerial 3), die Kappa-Koeffizienten sind dann nicht sinnvoll interpretierbar (Feinstein und Cicchetti 1990).

\section{Ergebnisse der Anwendung des Kategoriensystems auf die Schulbuchreihe Linder}

\section{Inhaltliche Befunde}

\section{Häufigkeit der Thematisierung von Energie}

Die Auszählung der Texte, Abbildungen und Aufgaben mit und ohne Energiebezug in stoffwechsel- und ökologiebezogenen Kapiteln zeigt, dass mit zunehmender Klassenstufe häufiger Energie thematisiert wird (vgl. Tab. 3). Allerdings wird Energie oft nur am Rande behandelt. In 38,8\% ( $n=$ 40) der analysierten Texte wird nicht mehr als einmal der Begriff Energie verwendet. In 68,5\% der Aufgaben wird Energie nur in der Musterlösung und nicht im Aufgabenstamm genannt.

\section{Definition von Energie}

Der Linder verzichtet in den untersuchten Kapiteln aller drei Bände und im Glossar des Gesamtbandes auf eine Definition von Energie. ${ }^{7}$

\section{Alternativvorstellungen}

Die Kategorie Alternativvorstellungen deckt zwei Aspekte ab. Erstens wurde die fachliche Korrektheit der dargestellten Inhalte überprüft. Besonderes Augenmerk lag auf der fachlich falschen Verwendung von Fachtermini und Aussagen oder Darstellungen, die Alternativvorstellungen der Schülerinnen und Schüler stützen oder erzeugen könnten, zum Beispiel die Verwendung des Begriffs Energie im Sinne der Alltagssprache. Als problematisch angesehen wurden 4,6\% der Texte/Abbildungen und 3,9\% der Aufgaben. Fachlich falsch ist beispielsweise die Darstellung des Energieflusses als Kreislauf. Indem Pfeile von den Destruenten und der Atmung zurück zu den Produzenten führen, werden energetische und stoffliche Aspekte vermischt (Linder Gesamtband, Abb. 389.3). Daneben wurden einige fachlich falsche Formulierungen festgestellt, z. B. „Wie gelingt es Pflanzen, Energie zu erzeugen?“"(Linder 2, S. 100). Zweitens wurde untersucht, ob aus der Forschung bekannte Alternativvorstellungen von Schülerinnen und Schülern aufgegriffen werden. Dies ist bei 1,8\% der Texte/Abbildungen und 3,1\% der Aufgaben der Fall, indem Alltags- und Fachsprache explizit unterschieden werden. Ein Beispiel ist die folgende Aufgabe aus dem Linder Gesamtband (S. 76): „In der Werbung für einen ,Energie-Drink' wird der folgende Slogan verwendet: ,X bringt verbrauchte Energie sofort zurück!' Nehmen Sie unter Verwendung der Fachsprache begründet Stellung." (vgl. auch Alternativvorstellung in Tab. 1).

\footnotetext{
7 Linder Biologie 1 und Linder Biologie 2 besitzen keine Glossare. Im Linder Gesamtband werden Komposita wie „Aktivierungsenergie“, „Freie Energie“ (als Synonym zur freien Enthalpie) und „Stoff- und Energieumwandlung“ erläutert.
} 
Abb. 1 Fließtextanalyse: Prozentuale Anteile der AnalyEnergieaspekte explizit berücksichtigen, Mehrfachzuordnungen waren möglich seeinheiten, die die einzelnen

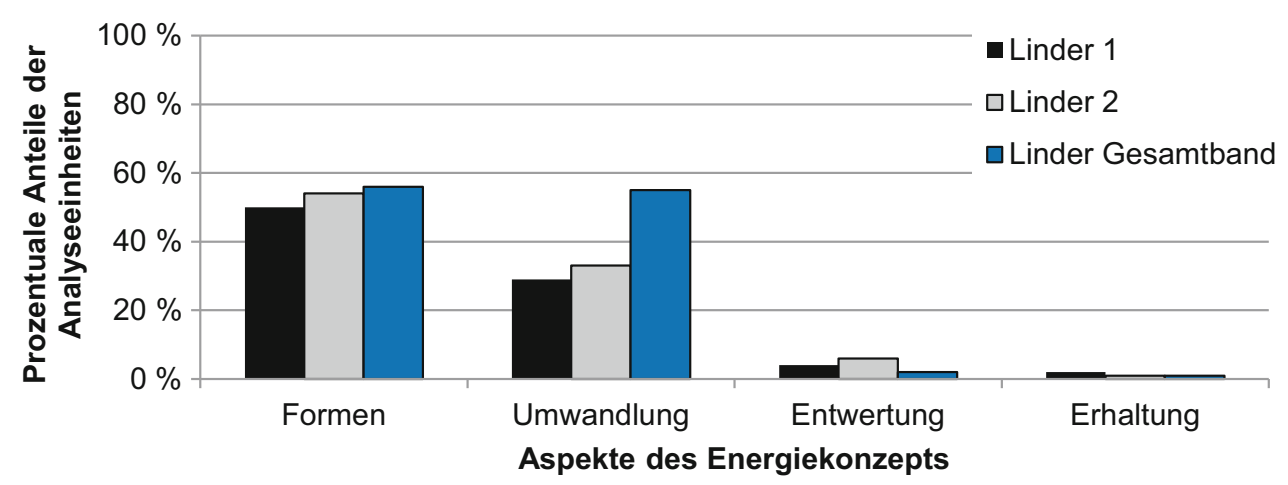

Abb. 2 Fließtextanalyse: Prozentuale Anteile der Analyseeinheiten, die die einzelnen Energieaspekte implizit berücksichtigen, Mehrfachzuordnungen waren möglich

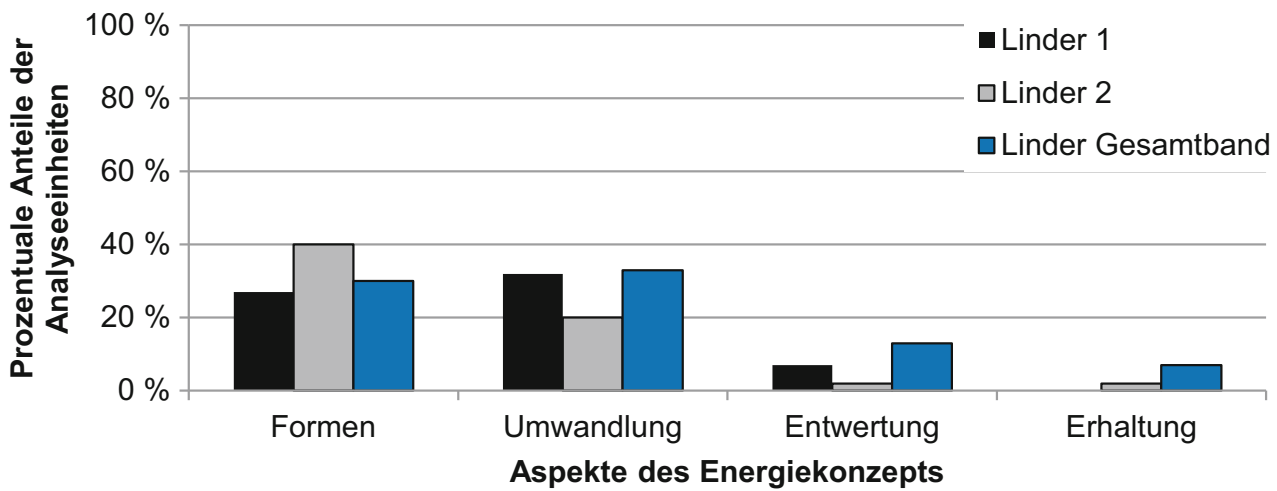

Thematisierte Aspekte des Energiekonzepts

Jede Repräsentation wurde dahingehend untersucht, welcher Aspekt des Energiekonzepts angesprochen wird, wobei Mehrfachzuordnungen möglich waren. Bei der Fließtextanalyse wurde zwischen expliziten und impliziten Thematisierungen unterschieden. Die Ergebnisse zeigen, dass Energieformen und -umwandlungen häufig, Energieentwertung und -erhaltung hingegen selten thematisiert werden. Dies gilt in allen Bänden (vgl. Abb. 1). Zusätzlich werden alle Energieaspekte implizit angesprochen (vgl. Abb. 2). Bei der Aufgabenanalyse wurden ausschließlich explizite Thematisierungen erfasst. Es ergibt sich ein ähnliches Bild wie bei der Fließtextanalyse: Energieentwertung und -erhaltung sind deutlich unterrepräsentiert (vgl. Abb. 3). Aufgrund des strengen Kriteriums für eine explizite Thematisierung der Energieentwertung wurden, wie bei der Fließtextanalyse, zusätzlich implizite Thematisierungen erfasst: 14,2 \% der Aufgaben behandeln Energieentwertung implizit, indem von einer Wärmeabgabe gesprochen wird.

Damit geht einher, dass das Energiekonzept selten in seiner Gesamtheit dargestellt wird. Nur 4,6\% $(n=13)$ der Texte und Abbildungen der Fließtextanalyse sprechen gleichzeitig alle vier Energieaspekte explizit oder implizit an. Werden nur die expliziten Thematisierungen berücksichtigt, verringert sich der Anteil auf 0,7\% ( $n=2$ Texte). Es gibt keine Aufgabe, die eine Auseinandersetzung mit allen vier Aspekten fordert.
Formale Befunde

Auszählung der Abbildungen

Während die Anzahl der Abbildungen pro Seite geringer wird, steigt der Anteil der Abbildungen mit Energiebezug vom Linder $1 \mathrm{zu} 2$ bis zu dem Gesamtband (vgl. Tab. 4).

\section{Abbildungstyp}

Der Linder verwendet eine große Bandbreite unterschiedlicher Abbildungstypen (vgl. Abb. 4). Einige Abbildungen sind Mischformen, das heißt, sie bestehen aus mehr als einem Typ, indem beispielsweise ein Flussdiagramm mit realistischen Zeichnungen innerhalb einer Abbildung kombiniert wird (vgl. z. B. Linder 2, S. 141, Weg der Energie).

Die Verteilung der verschiedenen Abbildungstypen unterscheidet sich jedoch zwischen Fließtext und Aufgaben. Während in den Unterkapiteln zahlreiche Fotos und realistische Zeichnungen vorkommen, werden bei Aufgaben mehr Tabellen und Achsendiagramme verwendet (vgl. Abb. 5).

Grundsätzlich lässt sich beschreiben, dass mit steigender Klassenstufe der Anteil an darstellenden Abbildungen (Fotos, realistische Zeichnungen) geringer und der Anteil an logisch-analytischen Abbildungen (Schemazeichnungen, Tabellen, Diagramme) höher ausfällt. Bei der Fließtextanalyse verringerte sich der Anteil darstellender Abbildungen von $67,4 \%$ in Linder 1 auf 24,0 \% im Linder Gesamtband, 
Abb. 3 Aufgabenanalyse: Prozentuale Anteile der Aufgaben, die die einzelnen Energieaspekte explizit berücksichtigen, Mehrfachzuordnungen waren möglich

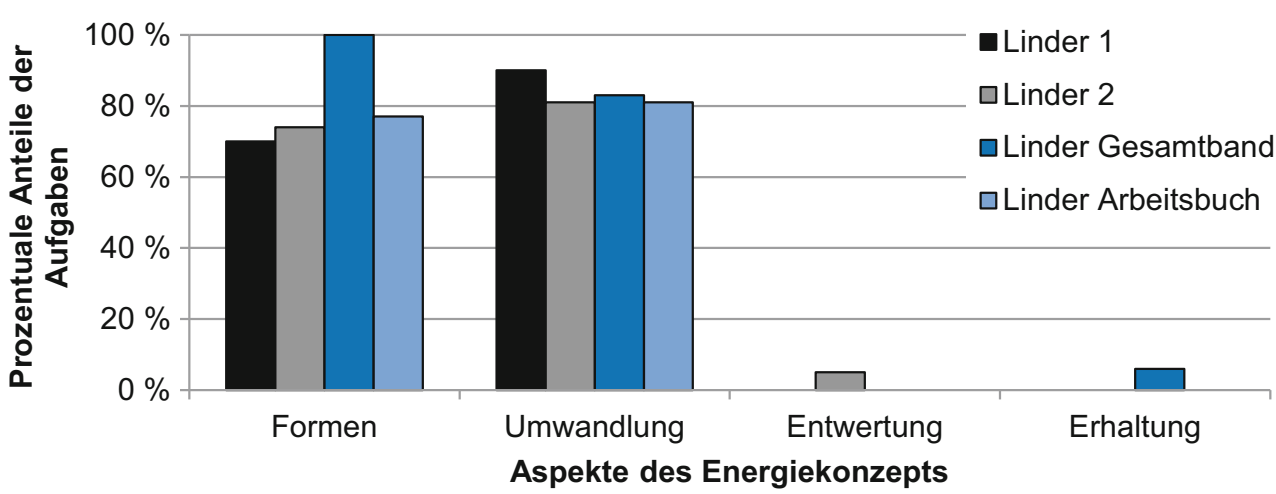

Tab. 4 Auszählung der Abbildungen in Kapiteln zu Stoffwechselbiologie und Ökologie

\begin{tabular}{lllll}
\hline & Linder 1 & Linder 2 & Linder Gesamtband & Linder Arbeitsbuch \\
\hline$\varnothing$ Anzahl Abbildungen pro Seite & 3,22 & 2,49 & 2,45 & - \\
$\varnothing$ Anzahl Abbildungen mit Energie- & 0,22 & 0,34 & 0,70 & - \\
$\begin{array}{l}\text { bezug pro Seite } \\
\varnothing \text { Anzahl Abbildungen pro Aufgabe }\end{array}$ & 0,70 & 0,64 & 0,72 & 1,26 \\
\hline
\end{tabular}

bei der Aufgabenanalyse von 47,4\% in Linder 1 auf durchschnittlich 18,7\% im Linder Gesamtband/Arbeitsbuch.

\section{Gestaltung der Abbildungen}

In dieser Kategorie wurden zwei zentrale Aspekte der Bildgestaltung erfasst: Die Verwendung von Untertiteln und Beschriftungen. Die Fließtextanalyse zeigt, dass 22,8\% der darstellenden und 97,5\% der logisch-analytischen Abbildungen eine Beschriftung aufweisen. Darüber hinaus sind 97,8\% der Abbildungen mit einem Untertitel versehen. Die Aufgabenanalyse ergab einen vergleichbaren Wert (95,5\% der Abbildungen mit Untertitel). Der Anteil an Abbildungen mit Beschriftung beträgt bei den Aufgaben 86,8\%. Dies hängt damit zusammen, dass sich Aufgaben wie bereits erläutert seltener auf darstellende Abbildungen beziehen.

\section{Funktion der Abbildung in Bezug auf den Text}

Es zeigt sich, dass die Abbildungen mit Organisations-/ Interpretationsfunktion in jeder Klassenstufe den größten Anteil ausmachen. Dieser Anteil wird mit steigender Klassenstufe größer (vgl. Abb. 6).

\section{Relation von Abbildung und Text}

Die Kategorie Relation zeigt den Grad der Redundanz von Bild- und Textinformation. Die Ergebnisse zeigen, dass schwach redundante Abbildungen mit steigender Klassenstufe häufiger vorkommen, während die stark redundanten und ergänzenden Abbildungen seltener vorkommen. Ergänzende Relationen sind selten (vgl. Abb. 7).
Die Relation ist eng verknüpft mit der Funktion der Abbildung: 77,3\% aller dekorativen und 61,9\% aller repräsentativen Abbildungen sind stark redundant; 75,2\% der Abbildungen mit Organisations-/Interpretationsfunktion sind schwach redundant. Abbildungen mit Zusatzfunktion sind in der Regel schwach redundant (50\%) oder ergänzend $(46,2 \%)$.

\section{Funktion der Abbildung für die Aufgabe}

Es zeigt sich, dass die meisten Abbildungen, die zu einer Aufgabe gehören, essentiell für deren Lösung sind: Die Funktion Informationsbereitstellung ist die häufigste in allen Bänden. Insgesamt erfüllen 86,7 \% der Abbildungen diese Funktion. Dekorative Abbildungen nehmen insgesamt einen Anteil von 9,7\% ein, wobei sie im Linder 1 mit 28,6\% deutlich häufiger eingesetzt werden. In seltenen Fällen dienen Abbildungen als Vorlage für das Erstellen einer eigenen Abbildung (3,5\%; z. B. Linder 2, S. 184, V1). ${ }^{8}$

\section{Anforderungen der Aufgabe}

Informationsentnahme: Die Informationsentnahme aus einem Text und/oder aus einer Abbildung wird in 69,3\% der Aufgaben explizit gefordert.

Konstruktion: Die Konstruktion einer Repräsentation wird in $11,0 \%$ der Aufgaben verlangt. Der Großteil davon $(9,4 \%)$ erfordert das Erstellen einer neuen Abbildung oder eines neuen Textes. Die übrigen 1,6\% der Aufgaben ( $n=2$, beide im Linder 2 ) erfordern das Ergänzen einer vorhandenen Abbildung (Tabelle).

\footnotetext{
8 Rundungsbedingt ergeben sich $99,9 \%$ anstatt $100 \%$.
} 
Abb. 4 Fließtextanalyse: Abbildungstypen, Mehrfachnennungen waren bei Mischformen möglich. Zur besseren Übersichtlichkeit wurden in der Kategorie Achsendiagramme Punktdiagramme, Kurven- oder Liniendiagramme und Säulendiagramme zusammengefasst. Flussdiagramme umfassen sowohl Sequenzen als auch Kreisläufe. Die Kategorie Sonstige beinhaltet Kreisdiagramme und spezielle biologische Diagramme wie z. B. Energiepyramiden

Abb. 5 Aufgabenanalyse: Abbildungstypen, auf die Bezug genommen wird, Mehrfachzuordnungen waren bei Mischformen möglich

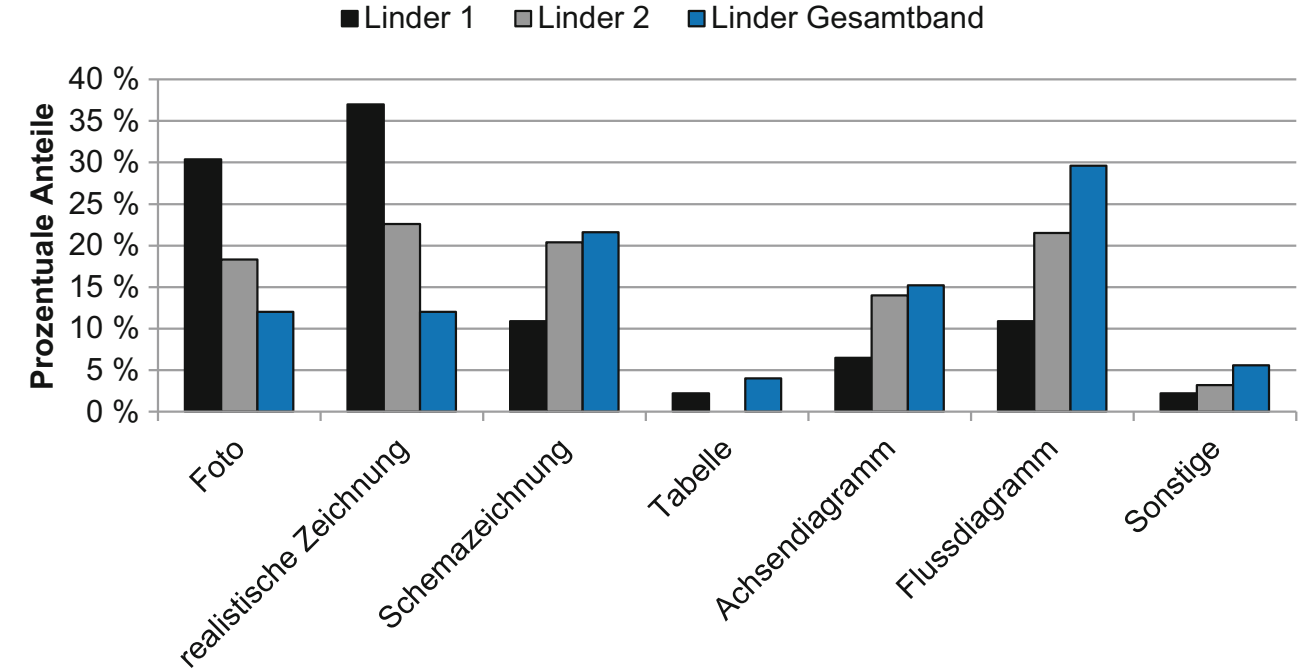

Abbildungstypen

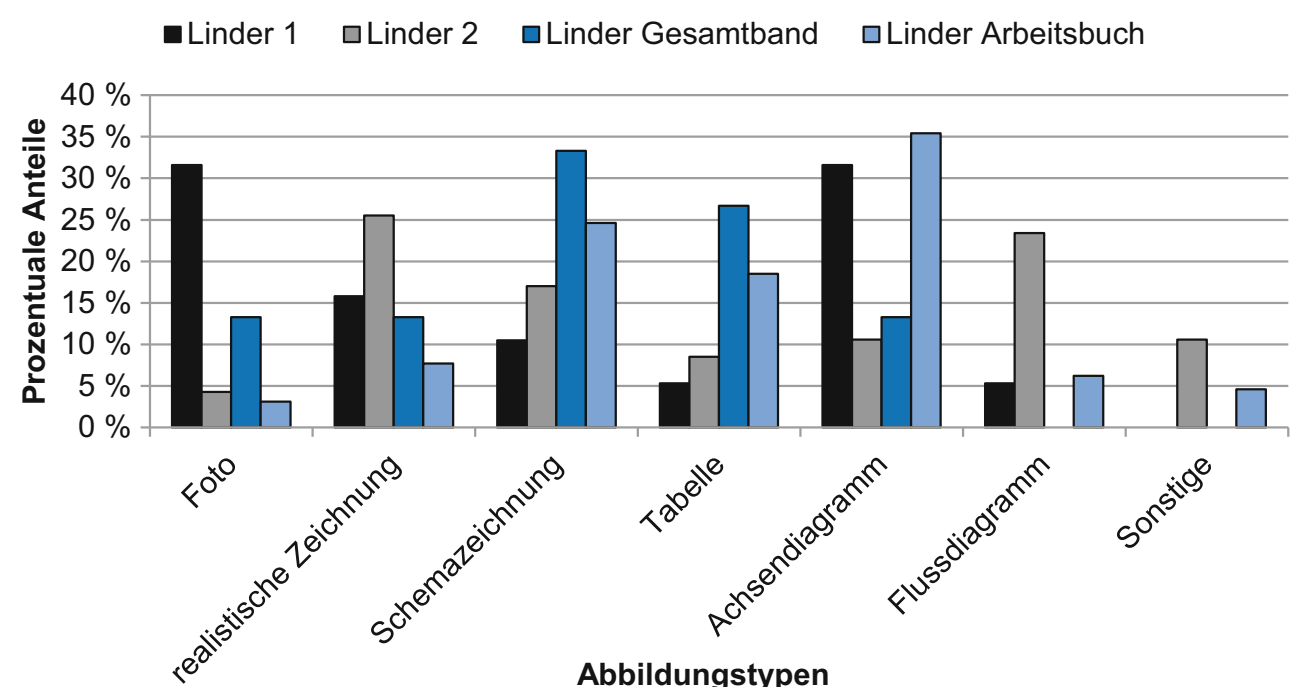

Integration: Bei 22,8\% aller Aufgaben müssen Informationen aus mehreren Repräsentationen miteinander verknüpft werden, um die Aufgabe zu lösen. Dies wird mit steigender Klassenstufe häufiger gefordert (von 10,0\% der Aufgaben in Linder 1 auf durchschnittlich 35,4\% in Linder Gesamtband und Arbeitsbuch). Vierzehn Aufgaben $(11,0 \%)$ verlangen die Verknüpfung von Informationen aus mehreren Abbildungen, zwei Aufgaben $(1,6 \%)$ aus mehreren Texten und dreizehn Aufgaben (10,2\%) aus Text und Abbildung.

Aufgaben zur Fachsprache: Alle Bände beinhalten Aufgaben, die die Lernenden zur Reflexion oder zur bewussten Verwendung von Fachsprache anhalten - ein Beispiel hierfür ist die Aufgabe zum Energie-Drink (vgl. Abschnitt ,Alternativvorstellungen"). Insgesamt betrifft dies $7,1 \%$ der Aufgaben.

\section{Diskussion}

\section{Diskussion der Ergebnisse}

Ziel der Studie war es, ein Kategoriensystem zu erstellen und exemplarisch anzuwenden, anhand dessen die Darstellung von Energie in Lehr-Lernmedien beschrieben werden kann. Das vorgestellte Kategoriensystem ist theoretisch fundiert und deckt sowohl inhaltliche als auch formale Aspekte der Darstellung ab. Im Rahmen des Interratings wurde eine zufriedenstellende Übereinstimmung zwischen den beiden Ratern erzielt, weshalb die Beschreibung der Kategorien als ausreichend ausgeschärft angesehen werden kann. $\mathrm{Zu}$ beachten ist, dass die angegebenen Kappa-Werte stichprobenspezifisch sind und in Folgestudien neu bestimmt werden müssen (Wirtz und Caspar 2002). Insbesondere die Kategorien, die nur sehr selten im Material beobachtet werden 
Abb. 6 Fließtextanalyse: Funktion der Abbildung in Bezug auf den Text, Organisations- und Interpretationsfunktion wurden zusammengefasst, Mehrfachzuordnungen waren nicht zugelassen

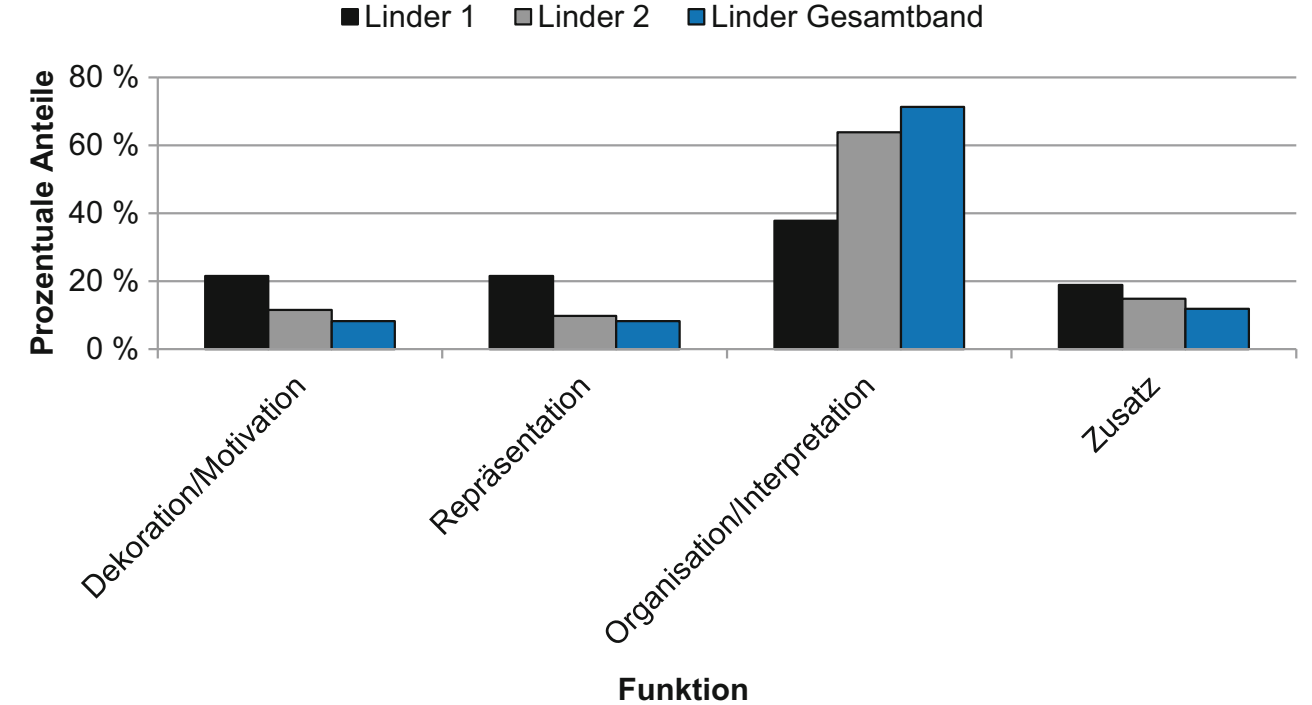

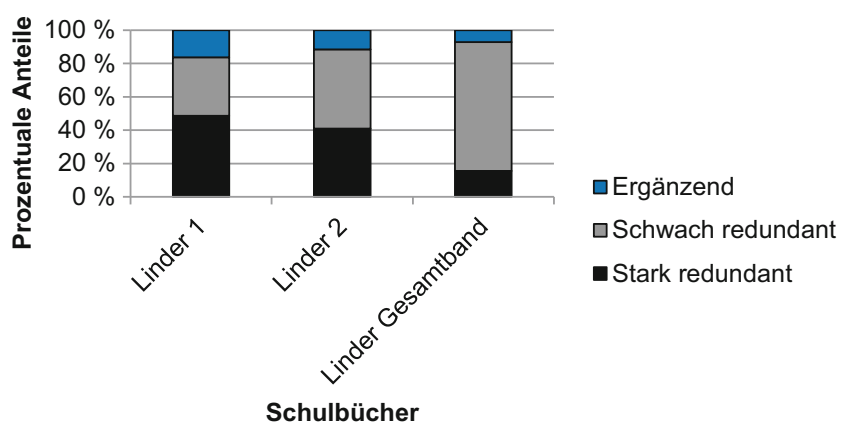

Abb. 7 Fließtextanalyse: Relation von Abbildung und Text

konnten, sollten dann kritisch überprüft werden. Neben der Anwendung des Kategoriensystems auf Biologieschulbücher ist auch eine Anwendung auf Chemie- und Physikschulbücher sowie auf andere Unterrichtsmedien denkbar.

Durch die Analyse der Schulbuchreihe Linder wurden exemplarisch erste Erkenntnisse über die Darstellung von Energie in deutschsprachigen Unterrichtsmedien gewonnen. Die Ergebnisse der inhaltsbezogenen Kategorien haben gezeigt, dass Energie mit steigender Klassenstufe häufiger thematisiert wird. Dieser Trend ist insofern erwartungskonform, als eine schrittweise Entwicklung des abstrakten Energiekonzeptes im Laufe der Klassenstufen sowohl empirisch belegt ist als auch normativ empfohlen wird (Neumann et al. 2013; Nordine 2016). Mit zunehmender Klassenstufe werden vermutlich häufiger komplexe energiebezogene Prozesse unterrichtet, während in der Unterstufe eher makroskopische Phänomene betrachtet werden. Beispielsweise zeigt sich im Biologielehrplan des bevölkerungsreichsten Bundeslandes Nordrhein-Westfalen, dass sich die basiskonzeptbezogenen Kompetenzen, die bis Ende der Klassenstufe 9 erreicht werden sollen, häufiger explizit auf Energie beziehen als die Kompetenzen, die bis
Ende der Klassenstufe 6 erreicht werden sollen (LP 2008). In Hinblick auf das Ergebnis des steigenden Energiebezugs muss jedoch berücksichtigt werden, dass oft nur implizit auf Energie eingegangen wird oder der Begriff Energie singulär auftritt. Dies ist nicht verwunderlich, da auch Abbildungen und Texte mit geringem Energiebezug in die Stichprobe aufgenommen wurden. Energie auch am Rande aufzugreifen kann die Relevanz und die Omnipräsenz des Konzeptes deutlich machen. Es ist jedoch wahrscheinlich, dass die tatsächliche Behandlung des Themas im Unterricht deutlich geringer ausfällt, als die Zahlen auf den ersten Blick vermuten lassen. Wird Energie beispielsweise nur in der Musterlösung und nicht im Aufgabenstamm genannt, wie es in fast $70 \%$ der untersuchten Aufgaben der Fall ist, ist fraglich, ob Lehrende und Lernende den Energiebezug erkennen und somit diese Lerngelegenheiten nutzen. Wie hoch der Energiebezug in der Praxis tatsächlich ausfällt, lässt sich nicht allein durch Schulbuchanalysen ermitteln, sondern würde Unterrichtsanalysen erfordern.

$\mathrm{Ob}$ es sinnvoll ist, Lernende mit einer Definition von Energie zu konfrontieren, ist umstritten, der Linder verzichtet in den untersuchten Kapiteln und im Glossar darauf. Positiv anzumerken ist, dass nur wenige fachliche Fehler festgestellt wurden. Auffallend war, dass Formulierungen wie „Energieverlust“ oder „Energie geht verloren“ im Linder häufig in Anführungsstriche gesetzt werden, ohne den Grund hierfür zu erklären. Dass Energie tatsächlich verloren gehen kann, stellt eine weit verbreitete Alternativvorstellung unter Schülerinnen und Schülern dar (Burger 2001), die möglicherweise durch die Formulierung in Schulbüchern - trotz der Verwendung von Anführungsstrichen begünstigt wird. Alternativvorstellungen über Energie werden nur in $2 \%$ der Texte und Abbildungen und in $3 \%$ der Aufgaben im Linder aufgegriffen. Gemäß der konstruktivistischen Lerntheorie sollten Alternativvorstellungen stär- 
ker explizit berücksichtigt werden. Konkrete Vorschläge, wie energiebezogene Alternativvorstellungen als Möglichkeit zum Umlernen genutzt werden können, bietet Kattmann (2015).

Die Analyse der Thematisierung der vier Aspekte des Energiekonzepts hat gezeigt, dass Energieerhaltung und Energieentwertung nur sehr selten thematisiert werden. Gerade bei diesen beiden Aspekten weisen Lernende die größten Verständnisschwierigkeiten auf (Jin und Anderson 2012). Vor allem der Aspekt Energieentwertung sollte häufiger explizit dargestellt werden, denn die bloße Schilderung einer Abgabe von Energie macht den Kern des Aspekts, nämlich den Verlust der Wertigkeit der Energie, für die Lernenden nicht deutlich. Nur mit einem Verständnis von Energieentwertung ist beispielsweise umweltbewusstes Handeln wie das „Sparen“ von Energie trotz des Grundsatzes der Energieerhaltung einsichtig (Lacy et al. 2014). Eine Darstellung aller vier Facetten in einer Repräsentation muss nicht wünschenswert sein, da sich eine inhaltliche Überfrachtung der einzelnen Repräsentation ergeben kann. Stattdessen können die Informationen aus verschiedenen Repräsentationen in einem gemeinsamen mentalen Modell integriert werden (Schnotz und Bannert 2003). Dieser Prozess kann durch Aufgaben, die die Lernenden zur Integration auffordern, unterstützt werden - vorausgesetzt, das Vorwissen der Lernenden ist ausreichend (Bodemer und Faust 2006). Detailliertere Analysen, beispielsweise in Hinblick auf das themenspezifisch notwendige Vorwissen oder die sachlogische Abhängigkeit einzelner Inhalte, könnten in Form von Sachstrukturanalysen erfolgen (z. B. Brückmann 2009; Wüsten et al. 2010).

Die Ergebnisse der formalen Kategorien sind weitestgehend positiv zu beurteilen. Ebenso wie in anderen Analysen zu Abbildungen in naturwissenschaftlichen bzw. biologischen Schulbüchern (Grundschule bis Sekundarstufe I: Dimopoulos et al. (2003); Sekundarstufe I bis II: Liu und Treagust (2013)) konnte beobachtet werden, dass darstellende Abbildungen mit steigender Klassenstufe seltener und logisch-analytische Abbildungen häufiger verwendet werden. Energie selbst ist nicht sichtbar, lediglich Energieträger und Umwandlungsprozesse können betrachtet werden. Deshalb sind logisch-analytische Abbildungen wie Graphen und Diagramme vermutlich eher dazu geeignet, das abstrakte Thema Energie darzustellen, könnten aber junge Lernende überfordern (Liu und Treagust 2013). Auffällig ist der vermehrte Gebrauch von Flussdiagrammen mit steigender Klassenstufe. Flussdiagramme werden vor allem zur Darstellung von Stoffwechselprozessen wie der Fotosynthese und zur Darstellung von Energieflüssen und Stoffkreisläufen eingesetzt, die vornehmlich in höheren Klassenstufen thematisiert werden. Die Beobachtung, dass bei Aufgaben vor allem auf Tabellen und Achsendiagramme Bezug genommen wird, könnte damit zusammenhängen, dass aus diesen Abbildungsformen komplexere Informationen entnommen werden können als aus darstellenden Abbildungen.

Eine hohe Redundanz von Text und Abbildung zeigt sich vor allem in unteren Klassenstufen. Dies entspricht den Empfehlungen der kognitionspsychologischen Forschung (Ainsworth 2006; Carney und Levin 2002). Dass Organisations- und Interpretationsfunktion von Abbildungen empirisch nicht getrennt werden konnten, liegt vermutlich an der nicht trennscharfen Definition der Funktionen und der Abhängigkeit vom Vorwissen der Lernenden (Levin et al. 1987). Nichtsdestoweniger konnte festgestellt werden, dass die Abbildungen größtenteils potenziell lernförderliche Funktionen besitzen und essentielle Informationen zum Lösen von Aufgaben bereitstellen. Die häufige Verwendung von Untertiteln ist positiv zu werten, ebenso wie die Verwendung von Beschriftungen, die vor allem bei logischanalytischen Abbildungen die Inhalte konkretisieren und den Fokus der Lernenden auf die wesentlichen Aspekte lenken.

Die Aufgaben könnten durch eine größere Variation in den Anforderungen verbessert werden. Positiv ist, dass die Informationsentnahme aus Texten und Abbildungen häufig gefordert wird. Integrationsaufgaben nehmen mit steigender Klassenstufe zu, was als sinnvoll erachtet werden kann, da bei dieser Fähigkeit eine Abhängigkeit vom Vorwissen und von der Vertrautheit mit den Abbildungstypen angenommen wird (Bodemer und Faust 2006). Dennoch könnte der Anteil solcher Aufgaben noch deutlich höher ausfallen. Darüber hinaus sollte die Konstruktion von Repräsentationen häufiger gefordert werden. Die Studie von Nitz et al. (2014) deutet darauf hin, dass sich die häufige Interpretation und seltene Eigenkonstruktion von Abbildungen, wie sie sich in der exemplarisch analysierten Schulbuchreihe zeigt, auch in der Unterrichtspraxis niederschlägt. Nichtsdestoweniger wird am Ende der Mittelstufe die Kompetenz gefordert, Informationen mit bildlichen Gestaltungsmitteln darzustellen. Ebenso wird die Anwendung fachbezogener Sprache erwartet (KMK 2004a). Im Linder ist der Anteil energiebezogener Aufgaben, die ausdrücklich auf die Verwendung von Fachsprache abzielen, jedoch gering. Gerade beim Thema Energie sollte die Differenz zwischen Alltagsund Fachsprache explizit gemacht werden, um sprachbedingte Alternativvorstellungen bewusst zu machen und so ein Verständnis des wissenschaftlich anerkannten Energiekonzepts zu fördern.

Dass die Schulbuchbände von unterschiedlichen Autorengruppen verfasst wurden, könnte eine alternative Erklärung für die beobachteten Unterschiede in der Darstellung sein. 


\section{Implikationen}

Lehrkräfte sollten in der Lage sein, das Unterrichtsmaterial vor dem Einsatz im Klassenraum selbstständig kritisch zu prüfen (Heitzmann und Niggli 2010). Bezüglich des Themas Energie können ihnen die hier vorgestellten Kriterien dafür Anhaltspunkte bieten. Die Ergebnisse der exemplarisch analysierten Schulbuchreihe zeigen potenzielle formale und inhaltliche Problembereiche von Unterrichtsmedien auf, denen in zukünftigen Untersuchungen besondere Beachtung geschenkt werden sollte. Wenn Lehrende ihren Unterricht auf Medien mit entsprechenden Problembereichen stützen, sollten sie diese durch didaktische Maßnahmen ausgleichen, indem sie beispielsweise Zusatzmaterialien hinzuziehen oder im Unterrichtsgespräch auf die vernachlässigten Aspekte des Energiekonzepts eingehen.

Zukünftige Forschungen sollten die Generalisierbarkeit der Ergebnisse anhand von Analysen weiterer Biologieschulbücher der Sekundarstufe I und II überprüfen. Interessant wäre zudem eine Überprüfung von Medien für die Hochschule. Angesichts der fächerübergreifenden Bedeutung des Energiekonzepts sollten außerdem Schulbücher der Chemie und Physik vergleichend betrachtet werden. Das hier vorgelegte Kategoriensystem könnte als Grundlage hierfür dienen und auf die jeweiligen Fächer adaptiert dafür herangezogen werden.

\section{Grenzen der Studie}

Ein Schwerpunkt wurde auf die Analyse von Abbildungen gelegt. Deshalb wurden weitere Eigenschaften der Darstellung, zum Beispiel die Textkohärenz, nicht berücksichtigt.

Offen bleibt, inwieweit die Ergebnisse für die Schulbuchreihe Linder auf andere Biologieschulbücher übertragbar sind. Außerdem wurde die Untersuchung auf Texte, Abbildungen und Aufgaben mit stoffwechselbiologischem bzw. ökologischem Bezug beschränkt.

In der durchgeführten Schulbuchanalyse wird ausschließlich der Angebotscharakter des Mediums betrachtet. Inwieweit dieses Angebot von den Lernenden erfolgreich genutzt wird, ist u. a. von den individuellen Fähigkeiten und von den kognitiven Anstrengungen der Lernenden in der konkreten Lernsituation abhängig (Helmke und Schrader 2014). Um die Nutzung der angebotenen Medien und den hieraus resultierenden Lernerfolg zu analysieren, werden entsprechende Lernstudien benötigt. Nichtsdestoweniger sollten Unterrichtsmedien so gut wie möglich gestaltet werden, um Lehrkräften qualitativ hochwertiges LehrLern-Material für den Unterricht zur Verfügung zu stellen. Das entwickelte Kategoriensystem kann hierzu einen Beitrag leisten.
Open Access Dieser Artikel wird unter der Creative Commons Namensnennung 4.0 International Lizenz (http://creativecommons.org/ licenses/by/4.0/deed.de) veröffentlicht, welche die Nutzung, Vervielfältigung, Bearbeitung, Verbreitung und Wiedergabe in jeglichem Medium und Format erlaubt, sofern Sie den/die ursprünglichen Autor(en) und die Quelle ordnungsgemäß nennen, einen Link zur Creative Commons Lizenz beifügen und angeben, ob Änderungen vorgenommen wurden.

\section{Literatur}

Ainsworth, S. (1999). The functions of multiple representations. Computers and Education, 33, 131-152. doi:10.1016/S03601315(99)00029-9.

Ainsworth, S. (2006). DeFT: A conceptual framework for considering learning with multiple representations. Learning and Instruction, 16, 183-198. doi:10.1016/j.learninstruc.2006.03.001.

Ametller, J., \& Pintó, R. (2002). Students' reading of innovative images of energy at secondary school level. International Journal of Science Education, 24, 285-312. doi:10.1080/ 09500690110078914.

Bayrhuber, H., Hauber, W., \& Kull, U. (Hrsg.). (2010). Linder Biologie Gesamtband. Braunschweig: Schroedel.

Bodemer, D., \& Faust, U. (2006). External and mental referencing of multiple representations. Computers in Human Behavior, 22, 27-42. doi:10.1016/j.chb.2005.01.005.

Brückmann, M. (2009). Sachstrukturen im Physikunterricht. Ergebnisse einer Videostudie. Berlin: Logos.

Burger, J. (2001). Schülervorstellungen zu „Energie im biologischen Kontext“. Ermittlungen, Analysen und Schlussfolgerungen. Dissertation, Universität Bielefeld.

Carney, R. N., \& Levin, J. R. (2002). Pictorial illustrations still improve students' learning from text. Educational Psychology Review, 14, 5-26. doi:10.1023/A:1013176309260.

Cohen, J. (1960). A coefficient of agreement for nominal scales. Educational and Psychological Measurement, 20, 37-46.

Cromley, J. G., Snyder-Hogan, L.E., \& Luciw-Dubas, U.A. (2010). Cognitive activities in complex science text and diagrams. Contemporary Educational Psychology, 35, 59-74. doi:10.1016/j. cedpsych.2009.10.002.

Dimopoulos, K., Koulaidis, V., \& Sklaveniti, S. (2003). Towards an analysis of visual images in school science textbooks and press articles about science and technology. Research in Science Education, 33, 189-216. doi:10.1023/A:1025006310503.

Duit, R. (1984). Learning the energy concept in school - empirical results from the Philippines and West Germany. Physics Education, 19, 59-66. doi:10.1088/0031-9120/19/2/306.

Duit, R. (2014). Teaching and learning the physics energy concept. In R.F. Chen, A. Eisenkraft, D. Fortus, J. Krajcik, K. Neumann, J. Nordine \& A. Scheff (Hrsg.), Teaching and learning of energy in K-12 education (S. 67-85). New York: Springer.

Erdmann, U., Jungbauer, W., \& Müller, M. (Hrsg.). (2008). Linder Biologie 1. Braunschweig: Schroedel.

Feinstein, A.R., \& Cicchetti, D. V. (1990). High agreement but low kappa: I. The problems of two paradoxes. Journal of Clinical Epidemiology, 43, 543-549. doi:10.1016/0895-4356(90)90158-L.

Feldermann, D. (Hrsg.). (2005). Linder Biologie Arbeitsbuch. Braunschweig: Schroedel.

Feynman, R. P., Leighton, R. B., \& Sands, M. (2007). Mechanik, Strahlung, Wärme. Feynman-Vorlesungen über Physik, Bd. 1. München: Oldenbourg.

Gropengießer, H. (2013). Schulbücher. In H. Gropengießer, U. Harms \& U. Kattmann (Hrsg.), Fachdidaktik Biologie (S. 390-394). Hallbergmoos: Aulis.

Härtig, H., Kauertz, A., \& Fischer, H.E. (2012). Das Schulbuch im Physikunterricht. Nutzung von Schulbüchern zur Unterrichtsvor- 
bereitung in Physik. Der mathematische und naturwissenschaftliche Unterricht, 65, 197-200.

Härtig, H., Bernholt, S., Prechtl, H., \& Retelsdorf, J. (2015). Unterrichtssprache im Fachunterricht - Stand der Forschung und Forschungsperspektiven am Beispiel des Textverständnisses. Zeitschrift für Didaktik der Naturwissenschaften, 21, 55-67. doi:10.1007/s40573-015-0027-7.

Heitzmann, A., \& Niggli, A. (2010). Lehrmittel - ihre Bedeutung für Bildungsprozesse und die Lehrerbildung. Beiträge zur Lehrerbildung, 28, 6-19.

Helmke, A., \& Schrader, F.-W. (2014). Angebot-Nutzungs-Modell der Wirkfaktoren pädagogischer Leistungen. In M. A. Wirtz (Hrsg.), Dorsch - Lexikon der Psychologie (S. 149-150). Bern: Huber.

Ibánez, M., \& Ramos, M. C. (2004). Physics textbooks presentation of the energy-conservation principle in hydrodynamics. Journal of Science Education and Technology, 13, 267-276.

Jin, H., \& Anderson, C.W. (2012). A learning progression for energy in socio-ecological systems. Journal of Research in Science Teaching, 49, 1149-1180. doi:10.1002/tea.21051.

Jin, H., \& Wei, X. (2014). Using ideas from the history of science and linguistics to develop a learning progression for energy in socioecological systems. In R. F. Chen, A. Eisenkraft, D. Fortus, J. Krajcik, K. Neumann, J. Nordine \& A. Scheff (Hrsg.), Teaching and learning of energy in K-12 education (S. 157-173). New York: Springer.

Kalyuga, S., Chandler, P., \& Sweller, J. (1998). Levels of expertise and instructional design. Human Factors, 40, 1-17. doi:10.1518/ 001872098779480587.

Kattmann, U. (2015). Schüler besser verstehen. Alltagsvorstellungen im Biologieunterricht. Hallbergmoos: Aulis.

KMK (2004a). Bildungsstandards im Fach Biologie für den Mittleren Schulabschluss. Beschluss vom 16.12.2004. https://www.kmk. org/fileadmin/Dateien/veroeffentlichungen_beschluesse/2004/ 2004_12_16-Bildungsstandards-Biologie.pdf. Zugegriffen: 15 . Mai 2014.

KMK (2004b). Bildungsstandards im Fach Physik für den Mittleren Schulabschluss. Beschluss vom 16.12.2004. https://www.kmk. org/fileadmin/Dateien/veroeffentlichungen_beschluesse/2004/ 2004_12_16-Bildungsstandards-Physik-Mittleren-SA.pdf. Zugegriffen: 07. Apr 2015.

KMK (2015). Übersicht zu Internetinformationen der Länder über zugelassene Lehr- und Lernmittel. http://www.kmk.org/fileadmin/ pdf/Bildung/AllgBildung/2015-04-14-Fu-Pf-Abfrage-LernmittelAnlage.pdf. Zugegriffen: 09. Dez 2015.

Konopka, H.-P., Paul, A., \& Starke, A. (Hrsg.). (2009). Linder Biologie 2. Braunschweig: Schroedel.

Lachmayer, S., Nerdel, C., \& Prechtl, H. (2007). Modellierung kognitiver Fähigkeiten beim Umgang mit Diagrammen im naturwissenschaftlichen Unterricht. Zeitschrift für Didaktik der Naturwissenschaften, 13, 145-160.

Lacy, S., Tobin, R. G., Wiser, M., \& Crissman, S. (2014). Looking through the energy lens: a proposed learning progression for energy in grades 3-5. In R. F. Chen, A. Eisenkraft, D. Fortus, J. Krajcik, K. Neumann, J. Nordine \& A. Scheff (Hrsg.), Teaching and learning of energy in K-12 education (S. 241-265). New York: Springer.

Landis, R.J., \& Koch, G.K. (1977). The measurement of observer agreement for categorical data. Biometrics, 33, 159-174. doi:10. $2307 / 2529310$.

Levin, J. R., Anglin, G. J., \& Carney, R. N. (1987). On empirically validating functions of pictures in prose. In D. M. Willows \& H. A. Houghton (Hrsg.), The psychology of illustration: basic research (S. 51-85). New York: Springer.

Liu, Y., \& Treagust, D.F. (2013). Content analysis of diagrams in secondary school science textbooks. In M. S. Khine (Hrsg.), Critical analysis of science textbooks. Evaluating instructional effectiveness (S. 287-300). Dordrecht: Springer.
LP 2008: Ministerium für Schule und Weiterbildung des Landes Nordrhein-Westfalen (2008). Kernlehrplan für das Gymnasium - Sekundarstufe I in Nordrhein-Westfalen. Biologie. http:// www.schulentwicklung.nrw.de/lehrplaene/upload/lehrplaene_ download/gymnasium_g8/gym8_biologie.pdf. Zugegriffen: 06 . Sep 2016.

Maier, U., Kleinknecht, M., Metz, K., \& Bohl, T. (2010). Ein allgemeindidaktisches Kategoriensystem zur Analyse des kognitiven Potentials von Aufgaben. Beiträge zur Lehrerbildung, 28, 84-96.

Mayer, R. E. (1993). Illustrations that instruct. In R. Glaser (Hrsg.), Advances in instructional psychology (Bd. 4, S. 253-284). Hillsdale: Lawrence Erlbaum Associates.

Mayer, R.E. (2011). Instruction based on visualizations. In R.E. Mayer \& P.A. Alexander (Hrsg.), Handbook of research on learning and instruction (S. 427-445). New York: Routledge.

McDonald, C. V. (2015). Evaluating junior secondary science textbook usage in Australian schools. Research in Science Education doi:10.1007/s11165-015-9468-8.

Neumann, K., Viering, T., Boone, W. J., \& Fischer, H.E. (2013). Towards a learning progression of energy. Journal of Research in Science Teaching, 50, 162-188. doi:10.1002/tea.21061.

Nitz, S., Nerdel, C., \& Prechtl, H. (2012). Entwicklung eines Erhebungsinstruments zur Erfassung der Verwendung von Fachsprache im Biologieunterricht. Zeitschrift für Didaktik der Naturwissenschaften, 18, 117-139.

Nitz, S., Ainsworth, S. E., Nerdel, C., \& Prechtl, H. (2014). Do student perceptions of teaching predict the development of representational competence and biological knowledge? Learning and Instruction, 31, 13-22. doi:10.1016/j.learninstruc.2013.12.003.

Nordine, J. (2016). What should students know about energy? In J. Nordine (Hrsg.), Teaching energy across the sciences $K-12$ (S. 17-38). Arlington: National Science Teachers Association.

Nordine, J., Krajcik, J., \& Fortus, D. (2011). Transforming energy instruction in middle school to support integrated understanding and future learning. Science Education, 95, 670-699. doi:10.1002/sce. 20423.

Opitz, S.T., Harms, U., Neumann, K., Kowalzik, K., \& Frank, A (2015). Students' energy concepts at the transition between primary and secondary school. Research in Science Education, 45, 691-715. doi:10.1007/s11165-014-9444-8.

Özkan, Ö., Tekkaya, C., \& Geban, Ö. (2004). Facilitating conceptual change in students' understanding of ecological concepts. Journal of Science Education and Technology, 13, 95-105. doi:10.1023/ B:JOST.0000019642.15673.a3.

Roth, W.-M., Bowen, G.M., \& McGinn, M.K. (1999). Differences in graph-related practices between high school biology textbooks and scientific ecology journals. Journal of Research in Science Teaching, 36, 977-1019. doi:10.1002/(SICI)10982736(199911)36:9〈977::AID-TEA3〉3.0.CO;2-V.

Schnotz, W. (1994). Wissenserwerb mit logischen Bildern. In B. Weidenmann (Hrsg.), Wissenserwerb mit Bildern (S. 95-147). Bern: Huber.

Schnotz, W. (2002). Commentary: Towards an integrated view of learning from text and visual displays. Educational Psychology Review, 14, 101-120. doi:10.1023/A:1013136727916.

Schnotz, W., \& Bannert, M. (2003). Construction and interference in learning from multiple representation. Learning and Instruction, 13, 141-156. doi:10.1016/s0959-4752(02)00017-8.

Slough, S. W., \& McTigue, E. (2013). Development of the graphical analysis protocol (GAP) for eliciting the graphical demands of science textbooks. In M.S. Khine (Hrsg.), Critical analysis of science textbooks. Evaluating instructional effectiveness (S. 17-30). Dordrecht: Springer.

Slough, S.W., McTigue, E.M., Kim, S., \& Jennings, S. K. (2010). Science textbooks' use of graphical representation: a descriptive analysis of four sixth grade science texts. Reading Psychology, 31, 301-325. doi:10.1080/02702710903256502. 
Stern, L., \& Roseman, J.E. (2004). Can middle-school science textbooks help students learn important ideas? Findings from project 2061's curriculum evaluation study: life science. Journal of Research in Science Teaching, 41, 538-568. doi:10.1002/tea.20019.

Sweller, J. (2005). The redundancy principle in multimedia learning. In R.E. Mayer (Hrsg.), The Cambridge handbook of multimedia learning (S. 159-167). New York: Cambridge University Press.

Trumper, R. (1991). Being constructive: an alternative approach to the teaching of the energy concept - part two. International Journal of Science Education, 13, 1-10. doi:10.1080/0950069910130101.

Tsui, C.-Y., \& Treagust, D.F. (2013). Introduction to multiple representations: their importance in biology and biological education. In D.F. Treagust \& C.-Y. Tsui (Hrsg.), Multiple representations in science education (S. 3-18). Dordrecht: Springer.
Wang, H.A. (1998). Science textbook studies reanalysis: Teachers "friendly" content analysis methods? Paper presented at the annual meeting of the National Association for Research In Science Teaching, San Diego. http://files.eric.ed.gov/fulltext/ED423142. pdf. Zugegriffen: 01. Sep 2016.

Weidenmann, B. (2004). Bilder zur Wissenskommunikation. In G. Reinmann \& H. Mandl (Hrsg.), Psychologie des Wissensmanagements. Perspektiven, Theorien und Methoden (S. 300-309). Göttingen: Hogrefe.

Wirtz, M., \& Caspar, F. (2002). Beurteilerübereinstimmung und Beurteilerreliabilität. Göttingen: Hogrefe.

Wüsten, S., Schmelzing, S., Sandmann, A., \& Neuhaus, B. (2010). Sachstrukturdiagramme - Eine Methode zur Erfassung inhaltsspezifischer Merkmale der Unterrichtsqualität im Biologieunterricht. Zeitschrift für Didaktik der Naturwissenschaften, 16, 23-39. 